\title{
Descripción de la resistencia de Helicobacter pylori a seis antibióticos de uso frecuente en Colombia
}

\author{
Helicobacter pylori susceptibility to six commonly used antibiotics in Colombia
}

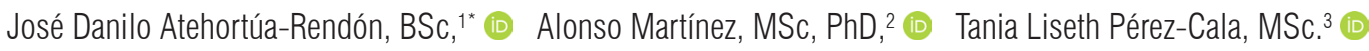

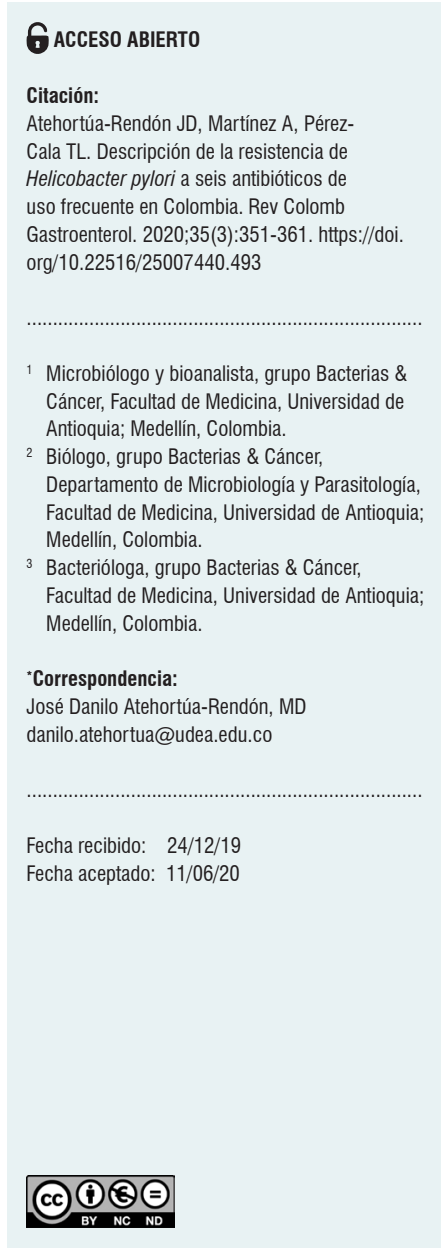

\begin{abstract}
Resumen
Helicobacter pylori (H. pylori) es un bacilo gramnegativo microaerófilo, capaz de colonizar la mucosa gástrica. Este microorganismo infecta a más de la mitad de la población mundial, por lo que se ha convertido en la infección bacteriana más común. La prevalencia de la infección y de las enfermedades asociadas a ella es alta, sobre todo en países en vías de desarrollo. El tratamiento recomendado para la erradicación es la triple terapia; sin embargo, su eficacia ha disminuido por el desconocimiento del patrón de susceptibilidad bacteriano por parte del personal médico y dada la aparición de cepas resistentes. La resistencia en $\mathrm{H}$. pylori se asocia con la capacidad de adaptación de la bacteria a ambientes hostiles y al uso de los antibióticos. En Colombia, existen reportes acerca de que $H$. pylori presenta resistencia a amoxicilina, metronidazol, claritromicina, furazolidona, levofloxacina y tetraciclina. Los estudios del patrón de susceptibilidad determinaron que la frecuencia de resistencia de $H$. pylori es variable y demuestran la falta de datos en la mayoría del territorio del país. Sobre la base de lo anterior, el objetivo de esta revisión es describir los porcentajes de resistencia de H. pylori a los antibióticos amoxicilina, metronidazol, claritromicina, furazolidona, levofloxacina y tetraciclina, usados en el tratamiento de la infección en los estudios realizados en Colombia.
\end{abstract}

\section{Palabras clave}

Helicobacter pylori, farmacorresistencia microbiana, enfermedades gastroduodenales, cáncer gástrico

\begin{abstract}
Helicobacter pylori (H. pylori) is a microaerophilic gram-negative bacillus that colonizes the gastric mucosa. It infects more than half the world's population, making it the most common bacterial infection. The prevalence of infection and associated diseases is high in developing countries. The recommended treatment for its eradication is triple therapy; however, its efficacy has decreased due to the lack of knowledge of the bacterial susceptibility pattern among the medical staff and the emergence of resistant strains. H. pylori susceptibility is associated with the bacteria's ability to adapt to hostile environments and the use of antibiotics. In Colombia, it has been reported that $H$. pylori is resistant to amoxicillin, metronidazole, clarithromycin, furazolidone, levofloxacin, and tetracycline. Studies on the susceptibility pattern have determined that the frequency of $H$. pylori susceptibility is variable and demonstrate the lack of data in most of the Colombian territory. With this in mind, the objective of this review is to describe the percentage of resistance to amoxicillin, metronidazole, clarithromycin, furazolidone, levofloxacin and tetracycline, which are used for the treatment of $H$. pylori infection, according to studies conducted in Colombia.
\end{abstract}

\section{Keywords}

Helicobacter pylori; Antimicrobial resistance; Gastroduodenal disorders; Gastric cancer. 


\section{INTRODUCCIÓN}

Helicobacter pylori ( $H$. pylori) es un bacilo gramnegativo, microaerófilo y pleomórfico, de aproximadamente 3,5 $\mu \mathrm{m}$ de longitud y $0,5 \mu \mathrm{m}$ de diámetro, que afecta alrededor de la mitad de la población mundial $(1,2)$. Los reportes de la infección varían, y se presentan prevalencias del 79,1\% en África, el $63,4 \%$ en Latinoamérica y el Caribe, el 54,7 \% en Asia, el $37,1 \%$ en Norteamérica y el $24,1 \%$ en Oceanía $(3,4)$.

Esta infección alcanza niveles más altos en países en desarrollo, dado que existe una relación bien establecida entre su aparición, el estatus socioeconómico y las condiciones de higiene (3, 5). La forma de transmisión no es clara, pero se plantea que se adquiere en la infancia por vía fecal-oral y es más frecuente de forma intrafamiliar (6). Otros estudios sugieren que también se obtiene por el consumo de agua o verduras contaminadas $(7,8)$.

En 1994, la Organización Mundial de la Salud (OMS), a través de la Agencia Internacional de Investigación sobre el Cáncer (International Agency for Research on Cancer, IARC) clasificó a H. pylori como un carcinógeno tipo I (9). La infección con este microorganismo es el principal factor de riesgo para el desarrollo de cáncer gástrico (CG), con una relación significativa positiva y un riesgo relativo del 3,8 (10). El CG causó la muerte de 782685 personas en el mundo en 2018 (10, 11).

Entre tanto, Colombia tiene una alta incidencia de infección por H. pylori (12). El manejo de esta se realiza sobre la base del consenso internacional de triple terapia (inhibidor de la bomba de protones, amoxicilina y claritromicina o metronidazol) (13). En nuestro país, en 2015, se publicó una guía para el tratamiento de las infecciones por $H$. pylori. Sin embargo, esta no se utiliza de manera generalizada por los médicos y debe ser ajustada de acuerdo con las tasas de resistencias locales (14).

La falla terapéutica (FT) en la erradicación de H. pylori es multifactorial e involucra factores genéticos, bacterianos y externos del paciente, como la no adherencia al esquema del tratamiento. Así, la principal causa de FT es la resistencia bacteriana a los antibióticos, adquirida durante el tratamiento (15). La resistencia a los antibióticos ocurre por la capacidad de los microrganismos para adaptarse de manera natural a medios hostiles, es intrínseca por la ausencia del sitio de unión del fármaco y adquirida por cambios genéticos. Además, $H$. pylori es capaz de lograr una diversidad genética por mutaciones y recombinaciones homólogas (16-23). La aparición de cepas resistentes también se asocia con el uso indiscriminado de antibióticos y la falta de adherencia al tratamiento como consecuencia de los efectos adversos de su utilización.

En la actualidad, en Colombia existen reportes de resistencia de $H$. pylori a amoxicilina, metronidazol, claritromi- cina, furazolidona, levofloxacina, moxifloxacina y tetraciclina (12, 24-29). Con todo ese contexto, el objetivo de esta revisión es describir los patrones de resistencia a dichos antibióticos (amoxicilina, metronidazol, claritromicina, furazolidona, levofloxacina y tetraciclina), los cuales han sido encontrados en el tratamiento de la infección por $H$. pylori durante los estudios realizados en el país.

\section{MATERIALES Y MÉTODOS}

\section{Estrategia de búsqueda y criterios de selección}

En las bases de datos PubMed (librería nacional de medicina de Estados Unidos, Bethesda, MD), LILACS (Literatura Latinoamericana de Información en Ciencias de la Salud: http://lilacs.bvsalud.org/en) y SciELO (librería científica electrónica: http://www.scielo.org), se realizó búsqueda de artículos con la estrategia: Helicobacter pylori, AND resistencia AND Colombia en español y Helicobacter pylori, AND drug resistance AND Colombia en inglés.

\section{Criterios de inclusión}

Se seleccionaron artículos originales, con textos completos, que presentaran datos de resistencia de H. pylori a antibióticos en Colombia, en cualquier año. De estos, se obtuvo información como el primer autor, el año de publicación, el lugar de realización, el año de toma de la muestra, los antibióticos evaluados, el número de muestras (biopsias gástricas o aislados de $H$. pylori), la prevalencia de la resistencia a antibióticos, el método de evaluación de la resistencia (prueba de epsilometría [E-test], difusión en disco o detección de mutaciones por reacción en cadena de la polimerasa [Polymerase Chain Reaction, PCR]).

\section{Criterios de exclusión}

Se excluyeron de la lista las revisiones de tema, los artículos que no permitían identificar la información necesaria y que estuviesen repetidos, además de los reportes que no abordaban el asunto de la resistencia de $H$. pylori al tratamiento con antibióticos.

\section{RESULTADOS}

La estrategia de búsqueda en español e inglés coincidió con 39 artículos. De estos, 23 cumplían con los criterios de inclusión. A continuación, se presenta un flujograma de los artículos incluidos en la revisión una vez aplicada la estrategia de búsqueda (Figura 1). Además, se presenta una tabla resumen con estudios de resistencia de $H$. pylori realizados en Colombia (Tabla 1). 


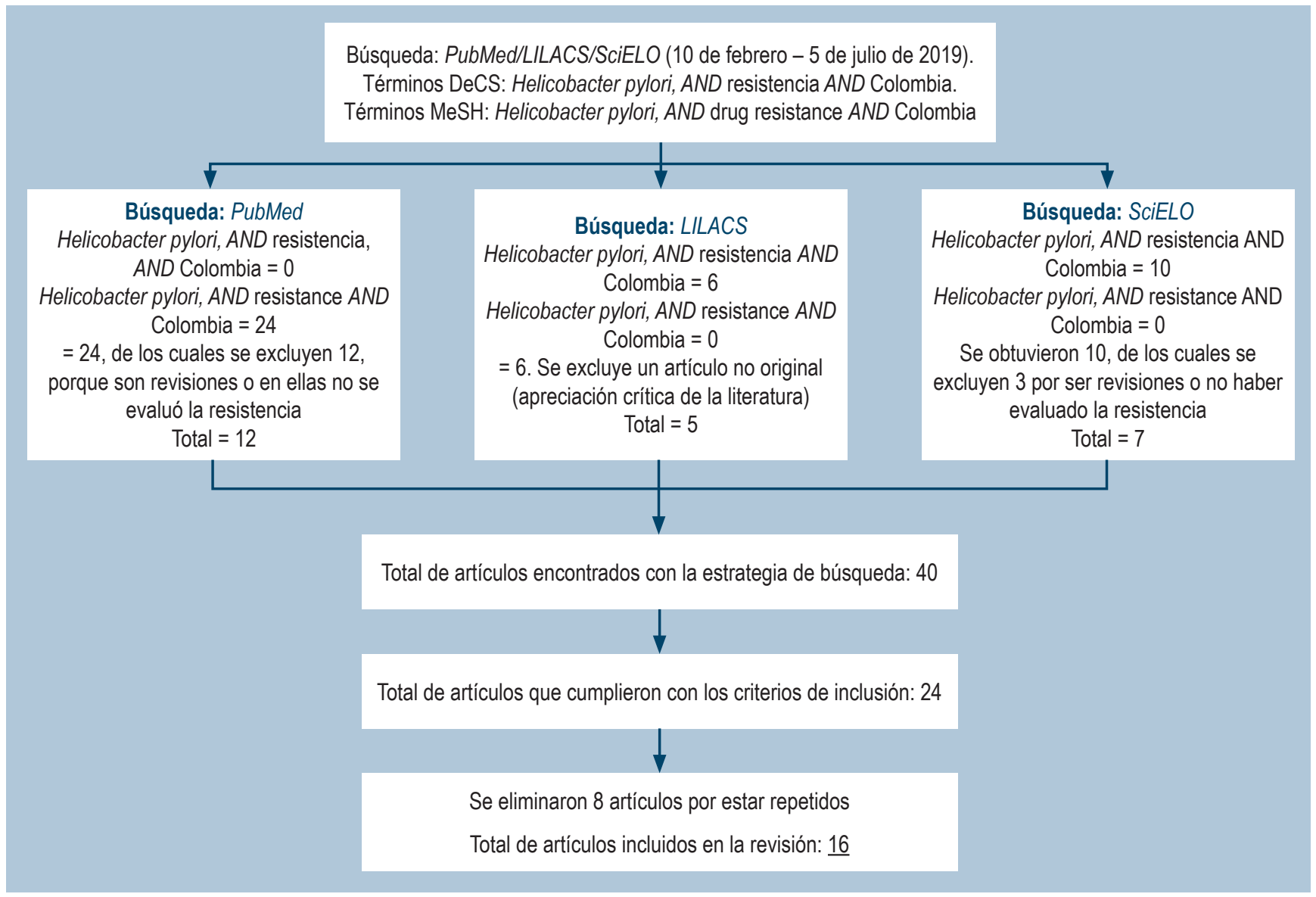

Figura 1. Esquema general de la estrategia de revisión sobre la resistencia de Helicobacter pylori a los antimicrobianos en Colombia. DeCS: descriptores en ciencias de la salud; MeSH: encabezados de temas médicos (Medical Subject Headings).

\section{Amoxicilina}

La amoxicilina es un antibiótico de primera elección para el tratamiento de H. pylori, dada la alta sensibilidad que presenta dicho microorganismo. Este fármaco es una penicilina semisintética que pertenece a los betalactámicos y es un inhibidor de la síntesis del peptidoglicano, ya que bloquea los transportadores denominados proteínas de unión a penicilina (Penicillin-Binding Protein, PBP) (13, 30). La bacteria adquiere resistencia a este medicamento por las mutaciones en el gen $p b p A(\mathrm{PBP})$, la producción de $\beta$-lactamasas y la presencia de bombas de eflujo. Los últimos dos aspectos se asocian con la resistencia más alta al medicamento $(25,31)$.

Así bien, las mutaciones más frecuentes en el gen $p b p A$ son sustituciones en posiciones específicas: en el aminoácido 556 ocurre una sustitución de una treonina por una serina (Thr556 $\rightarrow$ Ser), en la posición 648 se da un cambio de una lisina por una glicina (Lys648 $\rightarrow$ Gly), en la posi- ción 649 sucede un cambio de una arginina por una lisina (Arg649 $\rightarrow$ Lys) y en la posición 656 se observa un cambio de una arginina por una prolina (Arg656 $\rightarrow$ Pro).

Las primeras cepas de $H$. pylori resistentes a la amoxicilina se registraron en 1997, en un paciente que había sido tratado previamente (32). En 2009, se describió la aparición de una cepa productora de $\beta$-lactamasas (TEM-1), las cuales son capaces de destruir el anillo betalactámico del antibiótico (25). Además, se reportó la presencia de bombas de eflujo en la pared bacteriana, que expulsan el antibiótico hacia el exterior de la célula. Aunque esto solo se observó en cepas in vitro, es preocupante porque las bombas generan que las cepas se vuelvan multirresistentes (31).

En una revisión realizada en 2014 por Camargo y colaboradores, en la cual recopilaron los diferentes estudios de resistencia de H. pylori a los antibióticos en Latinoamérica, se evidenció que Colombia y Brasil presentaron las tasas de resistencia a la amoxicilina más altas, con 7 y $15 \%$, respectivamente (12). Entre tanto, en 2007, Gómez y cola- 
Tabla 1. Tabla resumen con estudios de resistencia de H. pylori realizados en Colombia

\begin{tabular}{|c|c|c|c|c|c|c|}
\hline Ciudades & Año & $\begin{array}{l}\text { Prueba } \\
\text { empleada }\end{array}$ & $\begin{array}{c}\text { Resistencia registrada } \\
\text { en porcentaje }(\%)\end{array}$ & $\begin{array}{l}\text { Mutación más } \\
\text { frecuente }\end{array}$ & Recomendaciones & Autores \\
\hline $\begin{array}{l}\text { Pereira, } \\
\text { Armenia y } \\
\text { Manizales }\end{array}$ & 2009 & $\begin{array}{l}\text { E-test } \\
\text { PCR }\end{array}$ & $\begin{array}{l}88 \% \text { : metronidazol, } \\
3,8 \% \text { : claritromicina }\end{array}$ & $\begin{array}{l}\text { A2143G ( } 82 \%) \\
\text { claritromicina }\end{array}$ & $\begin{array}{l}\text { No se debe incluir metronidazol en el tratamiento } \\
\text { empírico de la infección de } H \text {. pylori en esta región } \\
\text { para la población estudiada. }\end{array}$ & (30) \\
\hline $\begin{array}{l}\text { Pereira y } \\
\text { Armenia }\end{array}$ & 2009 & $\begin{array}{l}\text { E-test } \\
\text { PCR }\end{array}$ & $\begin{array}{l}88 \% \text { : metronidazol, } \\
2,2 \% \text { : claritromicina }\end{array}$ & $\begin{array}{l}\text { A2143G } \\
\text { claritromicina }\end{array}$ & $\begin{array}{l}\text { No se recomienda el uso de metronidazol y } \\
\text { claritromicina como primera línea de tratamiento. El } \\
50 \% \text { de las cepas puede tener doble resistencia en la } \\
\text { población estudiada. }\end{array}$ & (31) \\
\hline Bogotá & 2008 & $\begin{array}{l}\text { Difusión en } \\
\text { disco } \\
\text { E-test }\end{array}$ & $\begin{array}{l}93 \% \text { : metronidazol, } \\
60 \% \text { : claritromicina, } \\
86 \% \text { : tetraciclina, } \\
7 \% \text { : amoxicilina }\end{array}$ & No se evaluó & $\begin{array}{l}\text { De acuerdo con el consenso latinoamericano de } \\
\text { infecciones por } \mathrm{H} \text {. pylori, no se recomienda el uso } \\
\text { de metronidazol como tratamiento en la población } \\
\text { estudiada. }\end{array}$ & (32) \\
\hline Bogotá & 2009 & $\begin{array}{l}\text { E-test } \\
\text { Difusión en } \\
\text { disco }\end{array}$ & $\begin{array}{l}72 \% \text { : metronidazol, } \\
15 \% \text { : claritromicina }\end{array}$ & No se evaluó & $\begin{array}{l}\text { No se recomienda el uso de metronidazol en la } \\
\text { población estudiada. }\end{array}$ & (33) \\
\hline Bogotá & 2010 & E-test & $\begin{array}{l}81,01 \% \text { : metronidazol, } \\
17,72 \% \text { : claritromicina, } \\
3,8 \% \text { : amoxicilina }\end{array}$ & No se evaluó & $\begin{array}{l}\text { No se recomienda el uso de metronidazol o } \\
\text { claritromicina en la triple terapia como esquema de } \\
\text { primera línea en Bogotá. } \\
\text { La resistencia al metronidazol puede conducir a una } \\
\text { disminución de la eficacia del tratamiento en un } 50 \text { \% } \\
\text { en terapias triples y cuádruples. }\end{array}$ & $(34,35)$ \\
\hline Bogotá & 2013 & $\begin{array}{l}\text { Dilución en } \\
\text { agar } \\
\text { PCR }\end{array}$ & 13,6 \%: claritromicina & $\begin{array}{l}\text { A2143G } \\
\text { claritromicina } \\
(90,5 \%)\end{array}$ & $\begin{array}{l}\text { No se recomienda el uso de claritromicina en la triple } \\
\text { terapia como esquema de primera línea en la población } \\
\text { estudiada. }\end{array}$ & (36) \\
\hline Bogotá & 2016 & $\begin{array}{l}\text { Dilución en } \\
\text { agar } \\
\text { Secuenciación } \\
\text { directa }\end{array}$ & $27,3 \%$ : levofloxacina & $\begin{array}{l}\text { gyrA } \\
\text { mutación } \\
\text { N871 }\end{array}$ & $\begin{array}{l}\text { La resistencia a la levofloxacina viene incrementándose } \\
\text { en los últimos años y ha aumentado hasta el punto de } \\
\text { que no debe ser una elección aceptable para utilizarse } \\
\text { en tratamiento empírico en Bogotá. }\end{array}$ & (37) \\
\hline Bogotá & 2018 & PCR & $8 \%$ : claritromicina & 23SrDNA & $\begin{array}{l}\text { Estudio conducido en niños, debido a que la tasa de } \\
\text { resistencia a la claritromicina es }<15 \% \text {. Se sugiere } \\
\text { emplear en tratamiento convencional en niños. }\end{array}$ & (38) \\
\hline Bogotá & 2019 & $\begin{array}{l}\text { Dilución en } \\
\text { agar } \\
\text { Secuenciación } \\
\text { directa }\end{array}$ & $\begin{array}{l}\text { Datos de estudios } \\
\text { previos en Bogotá } \\
\text { 13,6\%: claritromicina, } \\
81,01 \% \text { : metronidazol, } \\
27,3 \% \text { : levofloxacina }\end{array}$ & $\begin{array}{l}\text { gyrA } \\
\text { Mutación } \\
\text { N871 }\end{array}$ & $\begin{array}{l}\text { No se recomienda el uso de metronidazol, } \\
\text { claritromicina o levofloxacina como tratamiento de la } \\
\text { infección por } H \text {. pylori en Bogotá. } \\
\text { Primer registro de aislamientos con resistencia múltiple. }\end{array}$ & (16) \\
\hline Bogotá & 2019 & PCR & $38,1 \%$ : claritromicina & $\begin{array}{l}\text { A2142G } \\
\text { claritromicina } \\
\text { A2143G }\end{array}$ & $\begin{array}{l}\text { La alta resistencia a la claritromicina (>15 \%) sugiere } \\
\text { reconsiderar su uso en tratamientos de erradicación en } \\
\text { la población estudiada. }\end{array}$ & (39) \\
\hline Antioquia & 2019 & PCR, RFLP & $\begin{array}{l}\text { 18,8 \%: } \\
\text { claritromicina }\end{array}$ & $\begin{array}{l}\text { A2142G } \\
\text { claritromicina } \\
\text { A2143G }\end{array}$ & $\begin{array}{l}\text { Primer registro de resistencia a los antibióticos en } \\
\text { Antioquia. }\end{array}$ & (40) \\
\hline Tumaco & 2012 & $\begin{array}{l}\text { Dilución en } \\
\text { agar }\end{array}$ & $\begin{array}{l}19,8 \% \text { : claritromicina, } \\
20,5 \% \text { : amoxicilina, } \\
\text { 10,96 \% fueron } \\
\text { resistentes a ambos }\end{array}$ & No se evaluó & $\begin{array}{l}\text { Primer registro de cepas multirresistentes a } \\
\text { claritromicina y amoxicilina. }\end{array}$ & (41) \\
\hline
\end{tabular}


Tabla 1. Tabla resumen con estudios de resistencia de H. pylori realizados en Colombia (continuación)

\begin{tabular}{|c|c|c|c|c|c|c|}
\hline Ciudades & Año & $\begin{array}{l}\text { Prueba } \\
\text { empleada }\end{array}$ & $\begin{array}{l}\text { Resistencia registrada } \\
\text { en porcentaje (\%) }\end{array}$ & $\begin{array}{l}\text { Mutación más } \\
\text { frecuente }\end{array}$ & Recomendaciones & Autores \\
\hline Túquerres & 2015 & $\begin{array}{l}\text { Dilución en } \\
\text { agar }\end{array}$ & $\begin{array}{l}4 \% \text { : claritromicina, } \\
2,7 \% \text { : amoxicilina }\end{array}$ & No se evaluó & $\begin{array}{l}\text { Estos antibióticos pueden ser usados libremente con } \\
\text { base en la poca resistencia presentada en la población } \\
\text { evaluada. }\end{array}$ & (42) \\
\hline Tumaco & 2018 & PCR & $19,8 \%$ : claritromicina & $\begin{array}{l}\text { A2143G }(69,8 \\
\%), A 2142 G \\
(11,7 \%) \text { y } \\
\text { A2142C }(2,6 \%)\end{array}$ & $\begin{array}{l}\text { Primer registro de cepas multirresistentes a } \\
\text { claritromicina y amoxicilina. }\end{array}$ & (43) \\
\hline Popayán & 2014 & $\begin{array}{l}\text { PCR } \\
\text { Secuenciación } \\
\text { directa }\end{array}$ & 4,3 \%: claritromicina & $\begin{array}{l}\text { A2143G } \\
\text { claritromicina }\end{array}$ & $\begin{array}{l}\text { La prevalencia de la resistencia de } \mathrm{H} \text {. pylori a la } \\
\text { claritromicina en la población de estudio indica que el } \\
\text { tratamiento triple estándar puede utilizarse de forma } \\
\text { empírica en la erradicación de la infección. }\end{array}$ & (44) \\
\hline Popayán & 2017 & $\begin{array}{l}\text { PCR } \\
\text { Secuenciación } \\
\text { directa }\end{array}$ & $\begin{array}{l}\text { No se evaluaron } \\
\text { aspectos fenotípicos }\end{array}$ & $\begin{array}{l}\text { RdxA } \\
\text { metronidazol } \\
(78 \%) \\
\text { D59N }\end{array}$ & $\begin{array}{l}\text { La frecuencia de mutaciones en la nitrorreductasa } \\
\text { RdxA en aislamientos de } H \text {. pylori, en Popayán, } \\
\text { sugiere que los tratamientos en los que se emplee el } \\
\text { metronidazol de manera empírica no serían una opción } \\
\text { válida para ser utilizada como terapia de erradicación } \\
\text { en pacientes de la población estudiada. }\end{array}$ & (45) \\
\hline
\end{tabular}

E-test: prueba de epsilometría; PCR: reacción en cadena de la polimerasa (Polymerase Chain Reaction); RFLP: fragmentos de restricción de longitud polimórfica (Restriction Fragment Length Polymorphisms)

boradores desarrollaron un estudio en el que encuestaron a 648 médicos (68\%: médicos generales, $19 \%$ : médicos internistas y $13 \%$ : gastroenterólogos) de 9 ciudades de Colombia. Luego de su análisis, los autores señalaron que la amoxicilina fue el antibiótico más prescrito para el tratamiento de $H$. pylori, con el 73 \% (33). En nuestro país, los estudios muestran tasas de resistencia de $H$. pylori a este antibiótico del 1,9, 3,8 y 9,5 \% (34-36).

\section{Metronidazol}

La acción bactericida del metronidazol depende de la reducción enzimática. En el caso de H. pylori, que no presenta la enzima superóxido dismutasa, la disminución del metronidazol es efectuada por la NADPH (nicotinamida adenina dinucleótido fosfato) nitrorreductasa y la $\mathrm{NAD}(\mathrm{P})$ $\mathrm{H}$ flavina oxidorreductasa, para producir radicales aniónicos como derivados nitrosos e hidroxilaminas, las cuales, al unirse al anillo imidazólico, conducen a una inhibición en la síntesis de los ácidos nucleicos (37-39). Los mecanismos de resistencia adquirida por la bacteria apuntan principalmente a mutaciones en el gen $R d x A$, que codifica la NADPH nitrorreductasa, y el $F d x B$, que codifica para la ferredoxina $(38,39)$.

Algunos autores afirman que las mutaciones puntuales en el $R d x A$ ocasionan la resistencia al metronidazol y, en menor frecuencia, los cambios en el FrxA. Mientras tanto, otros aseveran que las mutaciones del FrxA solo potencian la resistencia ocasionada por la inactivación del $R d x A$. Aunque se presentan mutaciones con la pérdida de función del $R d x A$, no todas las veces dicha pérdida de función implica una disminución de la susceptibilidad al medicamento, lo que conduce a que los resultados genotípicos obtenidos no sean concordantes con los in vitro e in vivo (40).

Además, los métodos de dilución en agar y la prueba de epsilometría presentan variabilidad inter e intraprueba y no son exactos, probablemente por el efecto de las condiciones medioambientales necesarias para el cultivo de la bacteria $(39,41)$. Respecto de la prueba de epsilometría, algunos autores sostienen que esta sobreestima la resistencia al metronidazol, por lo que se propone acompañar el diagnóstico de resistencia con pruebas de biología molecular.

En Latinoamérica, la resistencia al metronidazol reportada es $>30 \%$. Sin embargo, en países como Perú y Colombia el promedio documentado es del 66 y el $83 \%$, respectivamente (12). En nuestro país, diferentes estudios describen datos de resistencia a dicho medicamento, por métodos microbiológicos, que oscilan entre el 72 y el $97,6 \%$ (34-36). Asimismo, en 2017, Acosta y colaboradores evaluaron la frecuencia de mutaciones del $R d x A$ por métodos genotípicos y encontraron que el 78,2 \% de los casos $(133 / 170)$ portaban alguna alteración genética asociada 
con resistencia al fármaco (42). El metronidazol es uno de los antimicrobianos más prescritos por los médicos generales en Colombia para el tratamiento de H. pylori, con lo cual parecen ignorarse las altas tasas de resistencia (33).

\section{Claritromicina}

La claritromicina es un macrólido con actividad bacteriostática y bactericida, que inhibe la síntesis proteica al unirse al componente 23S de la subunidad ribosomal 50S (43). H. pylori adquiere resistencia a este antibiótico por mutaciones en la región peptidiltransferasa del domino $\mathrm{V}$ del gen HPrrnB23S, el cual codifica el componente ribosomal $23 S$ (43). Las mutaciones más frecuentes en este gen se localizan en las posiciones 2142, con la transición de una adenina por una guanina (A2142G) y la transversión adenina-citosina (A2142C). La transición de adenina por guanina en la posición 2143 (A2143G) genera un cambio conformacional en el sitio de unión del antibiótico e impide su acción $(44,45)$. Existen otras mutaciones de menor frecuencia como las transiciones T2182C (46), T2717C (47), G2224A, C2245T y T2289C (48).

Con respecto a Latinoamérica, se reportó una prevalencia de resistencia a los macrólidos del $14 \%$ en Argentina y del $13 \%$ en México (12). En Colombia, un estudio de 2009 realizado en el Eje Cafetero registró una baja resistencia a la claritromicina $(2,2 \%)$. Sin embargo, este medicamento se utiliza únicamente para el tratamiento de enfermedades respiratorias en la infancia, lo que explicaría la diferencia entre los porcentajes encontrados $(33,35)$.

Por otro lado, los estudios de prevalencia de la resistencia a los macrólidos en el país describen valores que oscilan entre el 13,6 y el $63,1 \%(29,34,36,49)$. En cuanto la resistencia genotípica, se ha determinado que la transición de adenina por guanina en la posición 2143 (A2143G) es la más frecuente (90,5\%), seguida por la transición A2142G $(7,1 \%)$ y la A2142C $(2,4 \%)(29)$.

\section{Furazolidona}

La furazolidona es uno de los nitrofuranos propuestos en el tratamiento de $\mathrm{H}$. pylori como terapia de rescate. Los nitrofuranos son bacteriostáticos, pero a altas dosis son bactericidas, y actúan de forma similar a los nitroimidazoles. El mecanismo de resistencia a este grupo de antibióticos es poco conocido. Se sugiere que las nitrorreductasas implicadas en este proceso son la flavodoxina piruvato oxidorreductasa (codificada por el gen PorCDAB) y la 2-oxoglutarate oxidorreductasa (OorDABC) (50).

$\mathrm{Su}$ y colaboradores identificaron mutaciones asociadas con la resistencia a la furazolidona en el gen $\operatorname{por} \mathrm{D}$, con transiciones de guanina por adenina en la posición 353 (G353A), adenina por guanina en la posición 356 (A356G) y citocina por timina en la posición 357 (C357T), y en oorD con las transiciones adenina (A041G, A122G) y la transversión (C349A). Sin embargo, algunas cepas resistentes al medicamento no portaban mutaciones, por lo que existe la posibilidad de otras mutaciones aún no determinadas (51). Las evidencias sugieren que otras enzimas activarían los nitrofuranos; no obstante, existen pocos estudios al respecto (52).

En Colombia, solo existe un estudio de resistencia fenotípica en $H$. pylori, el cual corresponde a un trabajo de grado que registró una resistencia del 4,8\%. Por lo demás, no hay estudios sobre resistencia genotípica a los nitrofuranos (33, 53). Por tanto, los nitrofuranos son de poca prescripción y se recomiendan como tratamiento de rescate en países como Brasil, en el que se ha descrito una resistencia del $3 \%$, similar a la encontrada en Bogotá (12).

\section{Levofloxacina}

La levofloxacina es una fluoroquinolona empleada en algunos casos como terapia de rescate en tratamientos no exitosos con claritromicina. Las fluoroquinolonas ejercen su acción al unirse a la subunidad $\mathrm{A}$ de la $\mathrm{ADN}$ girasa y evitan la formación del tetrámero (dos subunidades A y dos B), con lo cual bloquean la función de esta enzima. La subunidades A y B son codificadas por los genes gyrA y gyrB, respectivamente (37). La resistencia de $H$. pylori a las fluoroquinolonas se presenta por mutaciones en gyrA y gyrB y por alteraciones de las porinas.

Así, las mutaciones ocurren en la región determinante de resistencia a las quinolonas (Quinolone Resistance Domain Region, QRDR) del gyrA, lo que previene la unión del antibiótico a la girasa, al alterarse el sitio de unión de las quinolonas en el complejo ADN girasa-ADN (54). Las mutaciones descritas causantes de este fenómeno son mutaciones únicas: $\mathrm{Asn}_{87} \rightarrow \mathrm{Lys} \mathrm{Ala}_{88} \rightarrow \mathrm{Val}, \mathrm{Asp}_{91} \rightarrow$ Gly, Tyr o Asn, y mutaciones dobles en $\mathrm{Asp}_{91} \rightarrow$ Asn y en $\mathrm{Ala}_{88} \rightarrow \operatorname{Val}$ (55).

En Colombia, existe un estudio de seguimiento realizado en Bogotá, en el cual se evaluó la resistencia a la levofloxacina en biopsias provenientes de pacientes sometidos a endoscopia, entre 2009 y 2014. Este trabajo halló que, en 2009, la resistencia fue del 11,8\%, mientras que en 2014 fue del $27,3 \%$. Los autores consideraron que este aumento es resultado del uso de dicho medicamento en tratamientos para infecciones del tracto respiratorio y urinario (56).

\section{Tetraciclinas}

Las tetraciclinas son empleadas en los regímenes cuádruples para la erradicación de H. pylori (13). Estas presentan un efecto bacteriostático, al unirse de forma reversible a la 
subunidad ribosomal 30S, e inhiben la síntesis de proteínas. Las mutaciones descritas se hallan en el gen HPrnA$16 \mathrm{~S}$, que codifica para el componente $16 \mathrm{~S}$ de la subunidad menor (30S). Las más frecuentes involucran a los nucleótidos 926-928 (AGA $\rightarrow$ TTC). También se han registrado mutaciones dobles A926T/A928C, A926G/G927T, A926G/A928C y puntuales con la transición A926G o la transversión A939C (57-61).

En estudios realizados en Latinoamérica, se han reportado bajos valores de resistencia a la tetraciclina, que oscilan entre el 6 y el $14 \%$ (12). Sin embargo, en el caso de Colombia, Yepes y colaboradores documentaron en 2008 que el $85,7 \%(72 / 84)$ de las cepas aisladas en su estudio presentaban resistencia a dicho fármaco (34). Este dato difiere en gran medida con lo descrito en el continente.

\section{DISCUSIÓN}

De acuerdo con los porcentajes de resistencia del $H$. pylori en Colombia, el metronidazol se destaca como el antibiótico que causa mayor resistencia en la bacteria (Tabla $\mathbf{1}$ ). En Bogotá, los valores de resistencia registrados oscilan entre el 72 y el $93 \%(34,36,62)$, y en el Eje Cafetero el valor reportado llegó al 88 \% (35). Entre tanto, en Popayán se realizó el único estudio genotípico y se encontró que la mutación más frecuente fue en D59N (78 \%) (42). Los diferentes autores recomiendan no usar el metronidazol en el tratamiento de la infección por H. pylori en las ciudades del estudio. Por falta de más información, no es posible determinar la situación en el resto del país.

La claritromicina es el antibiótico con más estudios de resistencia en Colombia y muestra diferencias importantes. En el caso de Bogotá, los índices de resistencia a este medicamento han sido muy variables entre estudios: 13,6 y $60 \%$ (29, $34,36,62)$. Por su parte, en Tumaco el valor fue del 19,8 \% (63), y en Armenia y Pereira la resistencia fue más baja, pues alcanzó el 2,2 (35) y el 3,8 \% respectivamente (64).

En Popayán, el valor de resistencia genotípica a la claritromicina fue del 4,3 \%; la transición A2143G fue la más frecuente (65), mientras que en Antioquia fue del 18,8 \%, con una frecuencia del 81,5\% de la transición A2143G (49).

De igual forma, la resistencia a la amoxicilina se evaluó en 3 estudios; 2 de estos, realizados en Bogotá. En ellos se presentaron cifras del $7 \%$ en 2008 (34) y del 3,8 \% en 2010 (36). El tercer estudio se efectuó en Tumaco y encontró una resistencia del 20,5\% (63).

Con respecto a los valores de resistencia a la levofloxacina en Colombia, se encontró una cifra del 11,8 \% de las cepas estudiadas en Bogotá durante 2009, y del 27,3\%, en 2014 (56). Los autores sugieren que este medicamento

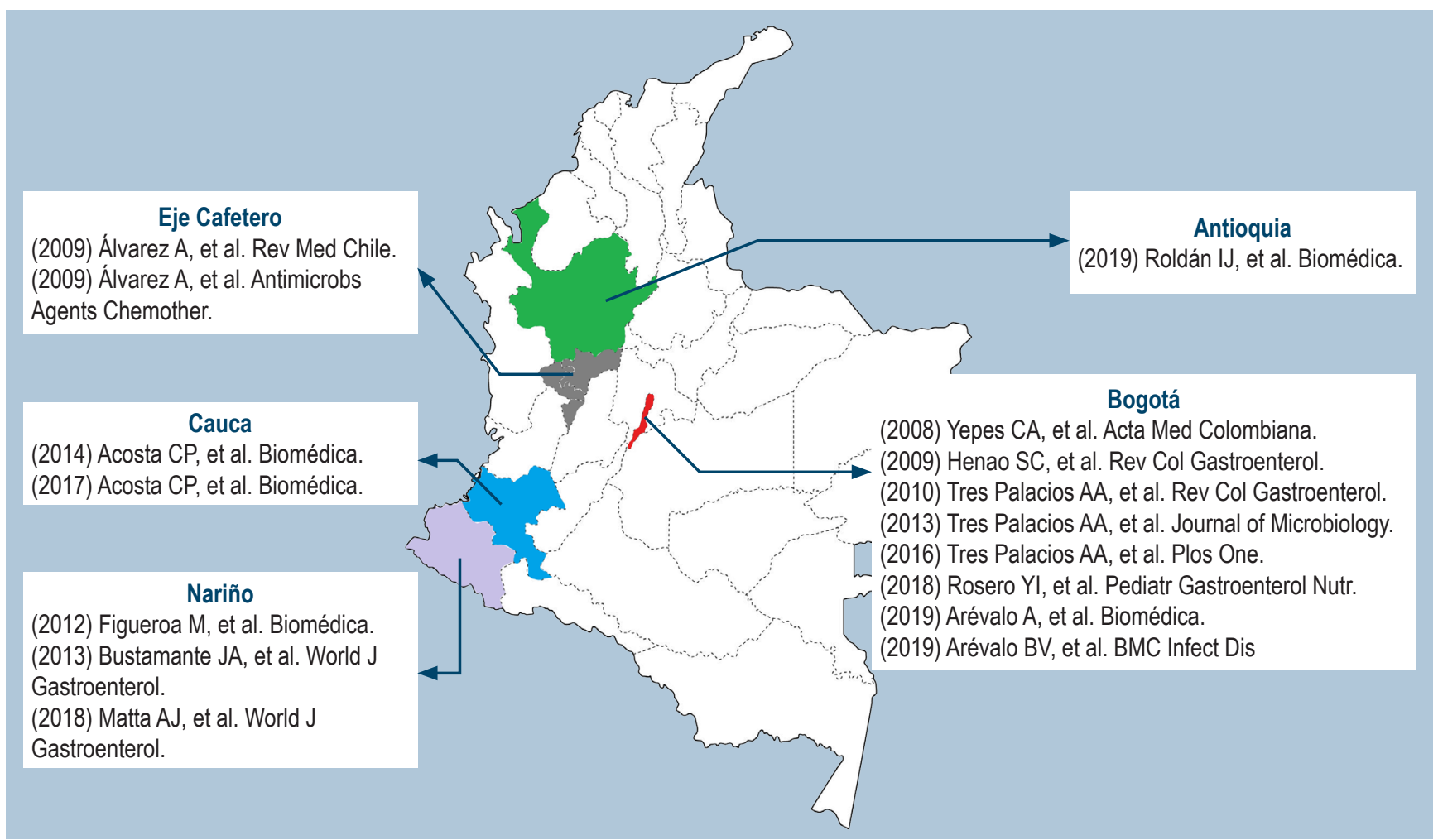

Figura 2. Mapa con estudios de resistencia de H. pylori realizados en Colombia. 
no debe ser considerado como un antibiótico de elección para el tratamiento empírico de la infección. Asimismo, la resistencia a la tetraciclina fue del $85,7 \%$. Dicho porcentaje proviene del único estudio que se ha desarrollado en nuestro país sobre la resistencia de $H$. pylori a este fármaco (34).

Por otra parte, 2 estudios han demostrado la aparición de cepas de $H$. pylori multirresistentes. El primero, realizado en Tumaco en 2012, detectó la presencia de cepas resistentes a la claritromicina y a la amoxicilina (63), mientras que el segundo, desarrollado por Arévalo y colaboradores en 2019, describió el hallazgo de cepas resistentes a 2 o más antibióticos (amoxicilina, claritromicina, levofloxacina y metronidazol). Estas cepas fueron provenientes de aislamientos de pacientes con 3 o 4 tratamientos fallidos (16).

Según la guía de práctica clínica para el diagnóstico y tratamiento de la infección por $H$. pylori en adultos, en Colombia se recomienda evitar la terapia triple combinada de amoxicilina, claritromicina y metronidazol o levofloxacina como tratamiento de primera línea, dado que existe evidencia que demuestra la resistencia primaria de la bacteria ante este tipo de manejo. Por tanto, se debe partir desde la epidemiología local para sugerir un tratamiento ajustado y adecuado, y evitar emplear antibióticos de manera empírica que puedan derivar en una falla terapéutica (14).
Los datos presentados en esta revisión muestran las variaciones existentes entre las poblaciones y señalan la importancia de determinar el patrón de resistencia de cada una. Esto, con el fin de permitir un seguimiento de la frecuencia con que se presentan las diferentes resistencias de las cepas circulantes en el país.

Es importante mencionar que los datos obtenidos en los estudios recopilados son aplicables solo a la población de estudio y no deben ser extrapolados al país en general. A continuación, se presenta un mapa, a manera de representación gráfica, de los estudios realizados en Colombia, y que fueron incluidos en esta revisión (Figura 2).

Así pues, los hallazgos de esta revisión señalan la necesidad de realizar más estudios que permitan determinar la resistencia de $H$. pylori en la población colombiana, a fin de definir los antibióticos que deben ser prescritos como primera línea o terapia de rescate.

\section{Fuente de financiación}

Colciencias. Código: 11157757202. Convocatoria 7772017 para proyectos de ciencia, tecnología e innovación en salud 2017.

\section{REFERENCIAS}

1. Loughlin MF. Novel therapeutic targets in Helicobacter pylori. Expert Opin Ther Targets. 2003;7(6):725-735. http://doi.org/10.1517/14728222.7.6.725

2. Kusters JG, van Vliet AH, Kuipers EJ. Pathogenesis of Helicobacter pylori infection. Clin Microbiol Rev. 2006;19(3):449-490. http://doi.org/10.1128/CMR.00054-05

3. O'Connor A, O’Morain CA, Ford AC. Population screening and treatment of Helicobacter pylori infection. Nat Rev Gastroenterol Hepatol. 2017; 14(4):230-240. http://doi.org/10.1038/nrgastro.2016.195

4. Silva GM, Silva HM, Nascimento J, Gonçalves JP, Pereira F, Lima R. Helicobacter pylori antimicrobial resistance in a pediatric population. Helicobacter. 2018;23(5):e12528. http://doi.org/10.1111/hel.12528

5. Nagy P, Johansson S, Molloy-Bland M. Systematic review of time trends in the prevalence of Helicobacter pylori infection in China and the USA. Gut Pathog. 2016;8:8. http://doi.org/10.1186/s13099-016-0091-7

6. Goh KL, Chan WK, Shiota S, Yamaoka Y. Epidemiology of Helicobacter pylori infection and public health implications. Helicobacter. 2011;16 Suppl 1(0 1):1-9. http://doi.org/10.1111/j.1523-5378.2011.00874.x
7. Fernández-Delgado M, Contreras M, García-Amado MA, Michelangeli F, Suárez P. Evidencias de la transmisión acuática de Helicobacter pylori. Interciencia. 2008;33(6):412417.

8. Gomes BC, de Martinis EC. Fate of Helicobacter pylori artificially inoculated in lettuce and carrot samples. Braz J Microbiol. 2004;35(1):145-150. https://doi.org/10.1590/S1517-83822004000100024

9. International Agency for Research on Cancer (IARC). Helicobacter pylori eradication as a strategy for preventing gastric cancer: IARC working group report, volume 8. Lyon: International Agency for Research on Cancer; 2014.

10. Bray F, Ferlay J, Soerjomataram I, Siegel RL, Torre LA, Jemal A. Global cancer statistics 2018: GLOBOCAN estimates of incidence and mortality worldwide for 36 cancers in 185 countries. CA Cancer J Clin. 2018;68(6):394-424. http://doi.org/10.3322/caac.21492

11. IARC Working Group on the Evaluation of Carcinogenic Risk to Humans. Schistosomes, Liver Flukes and Helicobacter pylori. Lyon (FR): International Agency for Research on Cancer; 1994. (IARC Monographs on the Evaluation of Carcinogenic Risks to Humans, No. 61.) Disponible en: https://www.ncbi.nlm.nih.gov/books/ NBK487782/ 
12. Camargo MC, García A, Riquelme A, Otero W, Camargo CA, Hernández-García T, Candia R, Bruce MG, Rabkin CS. The problem of Helicobacter pylori resistance to antibiotics: a systematic review in Latin America. Am J Gastroenterol. 2014;109(4):485-95. http://doi.org/10.1038/ajg.2014.24

13. Malfertheiner P, Megraud F, O’Morain CA, Gisbert JP, Kuipers EJ, Axon AT, Bazzoli F, Gasbarrini A, Atherton J, Graham DY, Hunt R, Moayyedi P, Rokkas T, Rugge M, Selgrad M, Suerbaum S, Sugano K, El-Omar EM; European Helicobacter and Microbiota Study Group and Consensus panel. Management of Helicobacter pylori infectionthe Maastricht V/Florence Consensus Report. Gut. 2017;66(1):6-30. http://doi.org/10.1136/gutjnl-2016-312288

14. Otero W, Trespalacios AA, Otero L, Vallejo MT, Torres M, Pardo R, Sabbagh L. Guía de práctica clínica para el diagnóstico y tratamiento de la infección por Helicobacter pylori en adultos. Rev Col Gastroenterol. 2015;30 supl. 1:17-33.

15. Wang D, Guo Q, Yuan Y, Gong Y. The antibiotic resistance of Helicobacter pylori to five antibiotics and influencing factors in an area of China with a high risk of gastric cancer. BMC Microbiol. 2019;19(1):152. http://doi.org/10.1186/s12866-019-1517-4

16. Arévalo A, Otero WA, Trespalacios AA. Helicobacter pylori: resistencia múltiple en pacientes de Bogotá, Colombia. Biomédica. 2019;39 supl. 1:125-134. https://doi.org/10.7705/biomedica.v39i3.4437

17. Björkholm B, Sjölund M, Falk PG, Berg OG, Engstrand L, Andersson DI. Mutation frequency and biological cost of antibiotic resistance in Helicobacter pylori. Proc Natl Acad Sci U S A. 2001;98(25):14607-14612. http://doi.org/10.1073/pnas.241517298

18. Jorgensen M, Daskalopoulos G, Warburton V, Mitchell HM, Hazell SL. Multiple strain colonization and metronidazole resistance in Helicobacter pylori-infected patients: identification from sequential and multiple biopsy specimens. J Infect Dis. 1996;174(3):631-635. http://doi.org/10.1093/infdis/174.3.631

19. Enroth H, Björkholm B, Engstrand L. Occurence of resistance mutation and clonal expansion in Helicobacter pylori multiple-strain infection: a potential risk in clarithromycinbased therapy. Clin Infect Dis. 1999;28(6):1305-1307. http://doi.org/10.1086/514796

20. Otero W, Trespalacios AA, Otero E. Helicobacter pylori: Tratamiento actual, un importante reto en gastroenterología. Rev Col Gastroenterol. 2009;24(3):279-292.

21. Furuta $Y$, Konno M, Osaki T, Yonezawa H, Ishige T, Imai M, Shiwa Y, Shibata-Hatta M, Kanesaki Y, Yoshikawa H, Kamiya S, Kobayashi I. Microevolution of VirulenceRelated Genes in Helicobacter pylori Familial Infection. PLoS One. 2015;10(5):e0127197. http://doi.org/10.1371/journal.pone.0127197

22. Cao Q, Didelot X, Wu Z, Li Z, He L, Li Y, Ni M, You Y, Lin X, Li Z, Gong Y, Zheng M, Zhang M, Liu J, Wang W,
Bo X, Falush D, Wang S, Zhang J. Progressive genomic convergence of two Helicobacter pylori strains during mixed infection of a patient with chronic gastritis. Gut. 2015;64(4):554-61. http://doi.org/10.1136/gutjnl-2014-307345

23. Suerbaum S, Smith JM, Bapumia K, Morelli G, Smith NH, Kunstmann E, Dyrek I, Achtman M. Free recombination within Helicobacter pylori. Proc Natl Acad Sci U S A. 1998;95(21):12619-24. http://doi.org/10.1073/pnas.95.21.12619

24. Andersson DI, Hughes D. Antibiotic resistance and its cost: is it possible to reverse resistance?. Nat Rev Microbiol. 2010;8(4):260-271. http://doi.org/10.1038/nrmicro2319

25. Tseng YS, Wu DC, Chang CY, Kuo CH, Yang YC, Jan CM, $\mathrm{Su}$ YC, Kuo FC, Chang LL. Amoxicillin resistance with beta-lactamase production in Helicobacter pylori. Eur J Clin Invest. 2009;39(9):807-12. http://doi.org/10.1111/j.1365-2362.2009.02166.x

26. Binh TT, Suzuki R, Trang T, Kwon DH, Yamaoka Y. Search for novel candidate mutations for metronidazole resistance in Helicobacter pylori using next-generation sequencing. Antimicrob Agents Chemother. 2015;59(4):2343-2348. http://doi.org/10.1128/AAC.04852-14

27. Wang LH, Cheng H, Hu FL, Li J. Distribution of gyrA mutations in fluoroquinolone-resistant Helicobacter pylori strains. World J Gastroenterol. 2010;16(18):2272-2277. http://doi.org/10.3748/wjg.v16.i18.2272

28. Talebi Bezmin Abadi A, Ghasemzadeh A, Taghvaei T, Mobarez AM. Primary resistance of Helicobacter pylori to levofloxacin and moxifloxacine in Iran. Intern Emerg Med. 2012;7(5):447-452. http://doi.org/10.1007/s11739-011-0563-1

29. Trespalacios AA, Otero W, Caminos JE, Mercado MM, Avila J, Rosero LE, Arévalo A, Poutou-Piñales RA, Graham DY. Phenotypic and genotypic analysis of clarithromycinresistant Helicobacter pylori from Bogotá D.C., Colombia. J Microbiol. 2013;51(4):448-52. http://doi.org/10.1007/s12275-013-2465-6

30. Mégraud F, Lehours P. Helicobacter pylori detection and antimicrobial susceptibility testing. Clin Microbiol Rev. 2007;20(2):280-322. http://doi.org/10.1128/CMR.00033-06

31. Liu ZQ Zheng PY, Yang PC. Efflux pump gene hefA of Helicobacter pylori plays an important role in multidrug resistance. World J Gastroenterol. 2008;14(33):5217-5222. http://doi.org/10.3748/wjg.14.5217

32. Dore MP, Sepúlveda AR, Mura I, Realdi G, Osato MS, Graham DY. Explanation for variability of omeprazole amoxycillin therapy? Tolerance of $\mathrm{H}$. pylori to amoxycillin. Gastroenterology.1997;112:A105.

33. Gómez M, Otero W, Gutiérrez O. Tratamiento de la infección por Helicobacter pylori. Encuesta en un grupo de médicos generales y especialistas en Colombia. Rev Col Gastroenterol. 2007;22(1):7-16.

34. Yepes CA, Rodríguez A, Ruiz A, Ariza B. Resistencia antibiótica del Helicobacter pylori en el Hospital Universitario 
San Ignacio de Bogotá. Acta Med Colomb. 2008;33(1):1114.

35. Álvarez A, Moncayo JI, Santacruz JJ, Corredor LF, Reinosa E, Martínez JW, Beltrán L. Resistencia a metronidazol y claritromicina en aislamientos de Helicobacter pylori de pacientes dispépticos en Colombia. Red Med Chile. 2009; $137(10): 1309-1314$. http://dx.doi.org/10.4067/S0034-98872009001000005

36. Trespalacios AA, Otero W, Mercado M. Resistencia de Helicobacter pylori a metronidazol, claritromicina y amoxicilina en pacientes colombianos. Rev Col Gastroenterol. 2010;25(1):31-38.

37. Francesco VD, Zullo A, Hassan C, Giorgio F, Rosania R, Ierardi E. Mechanisms of Helicobacter pylori antibiotic resistance: An updated appraisal. World J Gastrointest Pathophysiol. 2011;2(3):35-41. http://doi.org/10.4291/wjgp.v2.i3.35

38. Connor A, Vaira D, Gisbert JP, O’Morain C. Treatment of Helicobacter pylori Infection 2014. Helicobacter. 2014;19(1):38-45 https://doi.org/10.1111/hel.12163

39. Jenks PJ, Edwards DI. Metronidazole resistance in Helicobacter pylori. Int J Antimicrob Agents. 2002;19(1):1-7. http://doi.org/10.1016/s0924-8579(01)00468-x

40. Kim SY, Joo YM, Lee HS, Chung IS, Yoo YJ, Merrell DS, Cha JH. Genetic analysis of Helicobacter pylori clinical isolates suggests resistance to metronidazole can occur without the loss of functional rdxA. J Antibiot (Tokyo). 2009; 62(1):43-50. http://doi.org/10.1038/ja.2008.6

41. Osato MS, Reddy R, Reddy SG, Penland RL, Graham DY. Comparison of the Etest and the NCCLS-approved agar dilution method to detect metronidazole and clarithromycin resistant Helicobacter pylori. Int J Antimicrob Agents. 2001;17(1):39-44. http://doi.org/10.1016/s0924-8579(00)00320-4

42. Acosta CP, Quiroga AJ, Sierra CH, Trespalacios AA. Frecuencia de mutaciones de la nitrorreductasa $\mathrm{RdxA}$ de Helicobacter pylori para la activación del metronidazol en una población del departamento del Cauca, Colombia. Biomédica. 2017;37(2):191-199. https://doi.org/10.7705/biomedica.v37i2.3007

43. Versalovic J, Shortridge D, Kibler K, Griffy MV, Beyer J, Flamm RK, Tanaka SK, Graham DY, Go MF. Mutations in 23S rRNA are associated with clarithromycin resistance in Helicobacter pylori. Antimicrob Agents Chemother. 1996;40(2):477-80. http://doi.org/10.1128/AAC.40.2.477

44. Mégraud F, Corti R. Resistencia bacteriana del Helicobacter pylori en el mundo en el año 2009. Acta Gastroenterol Latinoam. 2009;39(4):282-290.

45. Schmitt BH, Regner M, Mangold KA, Thomson RB Jr, Kaul KL. PCR detection of clarithromycin-susceptible and -resistant Helicobacter pylori from formalin-fixed, paraffin-embedded gastric biopsies. Mod Pathol. 2013;26(9):1222-1227. http://doi.org/10.1038/modpathol.2013.48

46. Agudo S, Pérez-Pérez G, Alarcón T, López-Brea M. High prevalence of clarithromycin-resistant Helicobacter pylori strains and risk factors associated with resistance in Madrid, Spain. J Clin Microbiol. 2010;48(10):3703-3707. http://doi.org/10.1128/JCM.00144-10

47. Fontana C, Favaro M, Minelli S, Criscuolo AA, Pietroiusti A, Galante A, Favalli C. New site of modification of $23 \mathrm{~S}$ rRNA associated with clarithromycin resistance of Helicobacter pylori clinical isolates. Antimicrob Agents Chemother. 2002;46(12):3765-9. http://doi.org/10.1128/aac.46.12.3765-3769.2002

48. Hao Q, Li Y, Zhang ZJ, Liu Y, Gao H. New mutation points in $23 \mathrm{~S}$ rRNA gene associated with Helicobacter pylori resistance to clarithromycin in northeast China. World J Gastroenterol. 2004;10(7):1075-1077. http://doi.org/10.3748/wjg.v10.i7.1075

49. Roldán IJ, Castaño R, Navas MC. Mutaciones del gen ARN ribosómico 23S de Helicobacter pylori asociadas con resistencia a claritromicina en pacientes atendidos en una unidad de endoscopia de Medellín, Colombia. Biomédica. 2019;39(supl. 2):117-129. https://doi.org/10.7705/biomedica.v39i4.4377

50. Kwon DH, Lee M, Kim JJ, Kim JG, El-Zaatari FA, Osato MS, Graham DY. Furazolidone- and nitrofurantoinresistant Helicobacter pylori: prevalence and role of genes involved in metronidazole resistance. Antimicrob Agents Chemother. 2001;45(1):306-8. http://doi.org/10.1128/AAC.45.1.306-308.2001

51. Su Z, Xu H, Zhang C, Shao S, Li L, Wang H, Wang H, Qiu G. Mutations in Helicobacter pylori porD and oorD genes may contribute to furazolidone resistance. Croat Med J. 2006;47(3):410-5.

52. Sisson G, Goodwin A, Raudonikiene A, Hughes NJ, Mukhopadhyay AK, Berg DE, Hoffman PS. Enzymes associated with reductive activation and action of nitazoxanide, nitrofurans, and metronidazole in Helicobacter pylori. Antimicrob Agents Chemother. 2002;46(7):2116-23. http://doi.org/10.1128/aac.46.7.2116-2123.2002

53. Gambia CR, Quevedo NA. Determinación in vitro de la resistencia de Helicobacter pylori a la furazolidona. Bogotá: Facultad de Ciencias, Pontificia Universidad Javeriana; 2009.

54. Glocker E, Kist M. Rapid detection of point mutations in the gyrA gene of Helicobacter pylori conferring resistance to ciprofloxacin by a fluorescence resonance energy transfer-based real-time PCR approach. J Clin Microbiol. 2004;42(5):2241-2246. http://doi.org/10.1128/jcm.42.5.2241-2246.2004

55. Tankovic J, Lascols C, Sculo Q, Petit JC, Soussy CJ. Single and double mutations in gyrA but not in gyrB are associated with low- and high-level fluoroquinolone resistance in Helicobacter pylori. Antimicrob Agents Chemother. 2003;47(12):3942-3944. http://doi.org/10.1128/aac.47.12.3942-3944.2003 
56. Trespalacios-Rangel AA, Otero W, Arévalo-Galvis A, Poutou-Piñales RA, Rimbara E, Graham DY. Surveillance of Levofloxacin Resistance in Helicobacter pylori Isolates in Bogotá-Colombia (2009-2014). PLoS One. 2016;11(7):e0160007. http://doi.org/10.1371/journal.pone.0160007

57. Ribeiro ML, Gerrits MM, Benvengo YH, Berning M, Godoy AP, Kuipers EJ, Mendonça S, van Vliet AH, Pedrazzoli J Jr, Kusters JG. Detection of high-level tetracycline resistance in clinical isolates of Helicobacter pylori using PCR-RFLP. FEMS Immunol Med Microbiol. 2004;40(1):57-61. http://doi.org/10.1016/S0928-8244(03)00277-3

58. Gerrits MM, Berning M, Van Vliet AH, Kuipers EJ, Kusters JG. Effects of $16 \mathrm{~S}$ rRNA gene mutations on tetracycline resistance in Helicobacter pylori. Antimicrob Agents Chemother. 2003;47(9):2984-2986. http://doi.org/10.1128/aac.47.9.2984-2986.2003

59. Wu JY, Kim JJ, Reddy R, Wang WM, Graham DY, Kwon DH. Tetracycline-resistant clinical Helicobacter pylori isolates with and without mutations in $16 \mathrm{~S}$ rRNA-encoding genes. Antimicrob Agents Chemother. 2005;49(2):578-583. http://doi.org/10.1128/AAC.49.2.578-583.2005

60. Toledo H, López-Solís R. Tetracycline resistance in Chilean clinical isolates of Helicobacter pylori. J Antimicrob Chemother 2010;65(3):470-473. https://doi.org/10.1093/jac/dkp457

61. Lawson AJ, Elviss NC, Owen RJ. Real-time PCR detection and frequency of $16 \mathrm{~S}$ rDNA mutations associated with resistance and reduced susceptibility to tetracycline in Helicobacter pylori from England and Wales. J Antimicrob Chemother. 2005;56(2):282-286. http://doi.org/10.1093/jac/dki199

62. Henao SC, Otero W, Ángel LA, Martínez JD. Resistencia primaria a metronidazol en aislamientos de Helicobacter pylori en pacientes adultos de Bogotá, Colombia. Rev Col Gastroenterol. 2009;24(1):10-15.

63. Figueroa M, Cortés A, Pazos A, Bravo LE. Sensibilidad in vitro a amoxicilina y claritromicina de Helicobacter pylori obtenido de biopsias gástricas de pacientes en zona de bajo riesgo para cáncer gástrico. Biomédica. 2012;32(1):32-42. https://doi.org/10.7705/biomedica.v32i1.454

64. Álvarez A, Moncayo JI, Santacruz JJ, Santacoloma M, Corredor LF, Reinosa E. Antimicrobial susceptibility and mutations involved in clarithromycin resistance in Helicobacter pylori isolates from patients in the western central region of Colombia. Antimicrob Agents Chemother. 2009;53(9):4022-4024. http://doi.org/10.1128/AAC.00145-09

65. Acosta CP, Hurtado FA, Trespalacios AA. Determinación de mutaciones de un solo nucleótido en el gen $23 \mathrm{~S}$ rRNA de Helicobacter pylori relacionadas con resistencia a claritromicina en una población del departamento del Cauca, Colombia. Biomédica. 2014;34(Supl. 1):156-62. http://dx.doi.org/10.7705/biomedica.v34i0.1649 